\title{
Editorial
}

\section{Mental Health and COVID-19: How Aware Are We...?}

\author{
Pradeep K. Singh ${ }^{1} \quad$ Shraddha Singhania ${ }^{2, \bullet}$ Sohael M. Khan ${ }^{3, \oplus}$ \\ ${ }^{1}$ Department of Orthopaedics, Dr. L.H. Hiranandani Hospital, \\ Powai Mumbai, Maharashtra, India \\ 2Department of Radiodiagnosis, Acharya Vinoba Bhave Rural \\ Hospital, Wardha, Maharashtra, India \\ ${ }^{3}$ Department of Orthopaedics \& Spine Services, Acharya Vinoba \\ Bhave Rural Hospital, Wardha, Maharashtra, India \\ Int J Recent Surg Med Sci 2020;6:1-1
}

After World War II lasting from 1939 to 1945 which took drastic toll on count of the total world population, we now face COVID-19 pandemic that has caused a new catastrophe in the 20th century world. Everyone has already read up and soaked the knowledge of what is corona virus, what it does to the body, for which age group it is more harmful, and what we can do to prevent it.

Among the whole chaos there is an important aspect of health that has been forgotten or not paid attention to, and that is the mental health.

Unwillingly domesticated within our homes (or at places from where we cannot reach home) with free time, unlimited access to social media, news, and so on, people are looming and glooming over daily rise in number of cases of Coronavirus, limitations of the tests, unavailability of treatment, infringement on freedom, and growing financial losses. However, what we fail to understand is that slowly we are succumbing to poor mental health and are at high risk of emotional distress and depression.

We as health sector workers need to look into both aspects of the health care of the general public-that is, physical and mental health. As emotionally disturbed patients can exhibit unhealthy behavior such as substance abuse and noncooperation with health workers, they become dangerous to both themselves and the people around them. Besides the general population, doctors or health care providers are also at major risk to develop emotional instability due to indefinite long hours of work, inability to meet their loved ones, shortage in supply of protective gears, etc.

There should be proper measures taken in the form of webinars, posters, radio announcements, etc., to make people aware of importance of healthy mind. There can be check on social media regarding forwarding and circulation of fake news. Doctors can provide support through telecounseling and monitor psychosocial needs of their patients, and encourage healthy eating, meditation, etc. in their daily lifestyle. It is important to know that recovering from ill mental health is possible, all you need to do is-talk to someone, as Shannon L. Adler says:

As soon as "I" becomes "we," illness becomes wellness.

\section{Conflict of Interest}

None declared.
Address for correspondence Shraddha Singhania, MBBS, DMRD, Department of Radiodiagnosis, Acharya Vinoba Bhave Rural Hospital, Wardha, Maharashtra 442001, India (e-mail: shraddha2527@gmail.com).
DOI https://doi.org/ $10.1055 / \mathrm{s}-0040-1712708$ ISSN 2455-7420.
(C)2020 Medical and Surgical

Update Society
License terms

$(\circledast) \Theta \circledast$ 